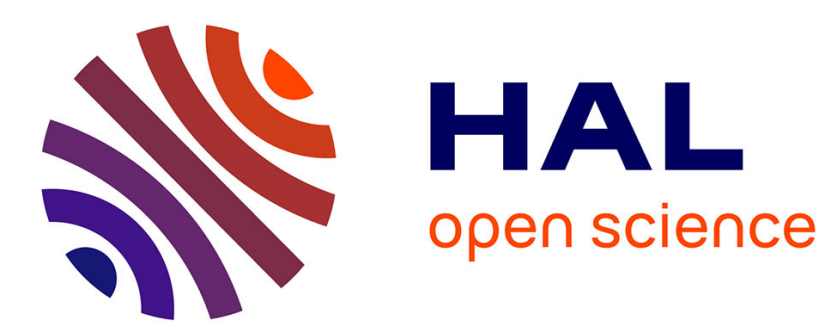

\title{
Déprise pastorale et dynamique des peuplements forestiers en montagne: l'exemple de Sansa, Pyrénées-Orientales
}

Bertrand Desailly

\section{- To cite this version:}

Bertrand Desailly. Déprise pastorale et dynamique des peuplements forestiers en montagne: l'exemple de Sansa, Pyrénées-Orientales. Revue Géographique des Pyrénées et du Sud-Ouest, 1988, 59 (1), pp.89-97. 10.3406/rgpso.1988.3109 . hal-03161063

\section{HAL Id: hal-03161063 \\ https://hal.science/hal-03161063}

Submitted on 5 Mar 2021

HAL is a multi-disciplinary open access archive for the deposit and dissemination of scientific research documents, whether they are published or not. The documents may come from teaching and research institutions in France or abroad, or from public or private research centers.
L'archive ouverte pluridisciplinaire HAL, est destinée au dépôt et à la diffusion de documents scientifiques de niveau recherche, publiés ou non, émanant des établissements d'enseignement et de recherche français ou étrangers, des laboratoires publics ou privés. 


\section{Déprise pastorale et dynamique des peuplements forestiers en} montagne : l'exemple de Sansa, Pyrénées-Orientales

M. Bertrand Desailly

\section{Citer ce document / Cite this document :}

Desailly Bertrand. Déprise pastorale et dynamique des peuplements forestiers en montagne : l'exemple de Sansa, PyrénéesOrientales. In: Revue géographique des Pyrénées et du Sud-Ouest, tome 59, fascicule 1, 1988. Montagnes fragiles. pp. 89-97; doi : https://doi.org/10.3406/rgpso.1988.3109

https://www.persee.fr/doc/rgpso_0035-3221_1988_num_59_1_3109

Fichier pdf généré le 06/04/2018 


\section{Résumé}

La soulane de Sansa (Pyrénées-Orientales) présente, entre $1400 \mathrm{~m}$ et $2370 \mathrm{~m}$, une juxtaposition de milieux à dynamiques forestières différentes, dans le temps comme dans l'espace. La combinaison de facteurs physiques (histoire du climat) et humains (pratiques pastorales) permet d'expliquer les variations dans le repeuplement forestier.

\section{Abstract}

Pastoral abandonment and tree-growing dynamics. The Sansa « soulane " (south-facing slope) in the Pyrénées-Orientales, displays, between the altitudes of $1400 \mathrm{~m}$ and $2370 \mathrm{~m}$, a juxtaposition of environment with different forest dynamics in both time and space. The combination of physical factors (climatic history) and human factors (pastoral farming methods) gives an explanation of the changing pattern of reforestation.

\section{Resumen}

Retroceso del pastoreo y dinámica de las poblaciones forestales. La solana de Sansa (PyrénéesOrientales) presenta, entre 1400 y 2370 m, una yuxtaposición de medios naturales caracterizados por dinámicas forestales diversas, tanto en el tiempo como en el espacio. La combinación de factores físicos (historia del clima) y humanos (prácticas pastorales) permiten entender las variaciones observables en la repoblación forestal. 
REVUE GÉOGRAPHIQUE DES PYRÉNÉES ET DU SUD-OUEST TOME 59, FASC. 1, pp. 89-97, Toulouse, 1988.

\title{
Déprise pastorale et dynamique des peuplements forestiers en montagne : l'exemple de Sansa, Pyrénées-Orientales :
}

\author{
par Bertrand DESAILLY *
}

\begin{abstract}
Le texte qui suit présente succinctement quelques résultats d'une étude entreprise au cours de l'année 1983-1984, dans le cadre des activités du Centre de Géographie physique Henri Elhai de l'Université de Paris X-Nanterre. Il s'agissait d'apprécier les transformations du paysage au cours des deux derniers siècles dans une commune du département des Pyrénées-Orientales, située en zone de montagne: Sansa. Outre de nombreuses observations effectuées directement sur le terrain, la consultation des archives municipales, des fonds de l'Office national des Forêts, la comparaison enfin de couvertures photographiques aériennes d'âges différents nous a permis de mener à bien ce travail.

Notre attention s'est naturellement portée sur la dynamique historique des peuplements arborés. Soumis à des contraintes de tout ordre (climatiques, anthropiques), le couvert végétal se modifie rapidement. Il faisait peu de doute que le bouleversement socio-économique des cent dernières années qui, ici comme ailleurs, fit s'effondrer la population et diminuer considérablement la pression pastorale, avait eu des conséquences directes sur l'emprise de l'arbre à l'intérieur du territoire communal. Ces effets restaient à préciser, quitte à dépasser quelques idées répandues, par exemple que la déprise pastorale implique seule et nécessairement une importante reforestation naturelle.
\end{abstract}

(*) Agrégé de géographie, assistant à l’Université de Toulouse-Le Mirail, et groupe "PARC " (Paysages : approche, recherches, comparaisons), Ecole normale supérieure de Saint-Cloud-Fontenay), 92211 Saint-Cloud Cedex. 


\section{Un ancien versant pastoral dans une commune vidée de ses habitants.}

La commune de Sansa se tient sur le versant sud du Massif du Madrès, au nord de la vallée de la Têt qui constitue l'épine dorsale du département des Pyrénées-Orientales. Elle appartient au Haut Conflent, région de relief escarpé, où se combinent les influences climatiques méditerranéennes et montagnardes. Le village offre à plus d'un titre l'aspect d'un bout du monde : une situation à l'extrémité amont d'une vallée affluente de la Têt, un accès malaisé, une population réduite à six habitants au recensement de 1982. La fréquentation touristique estivale demeure aujourd'hui très limitée.

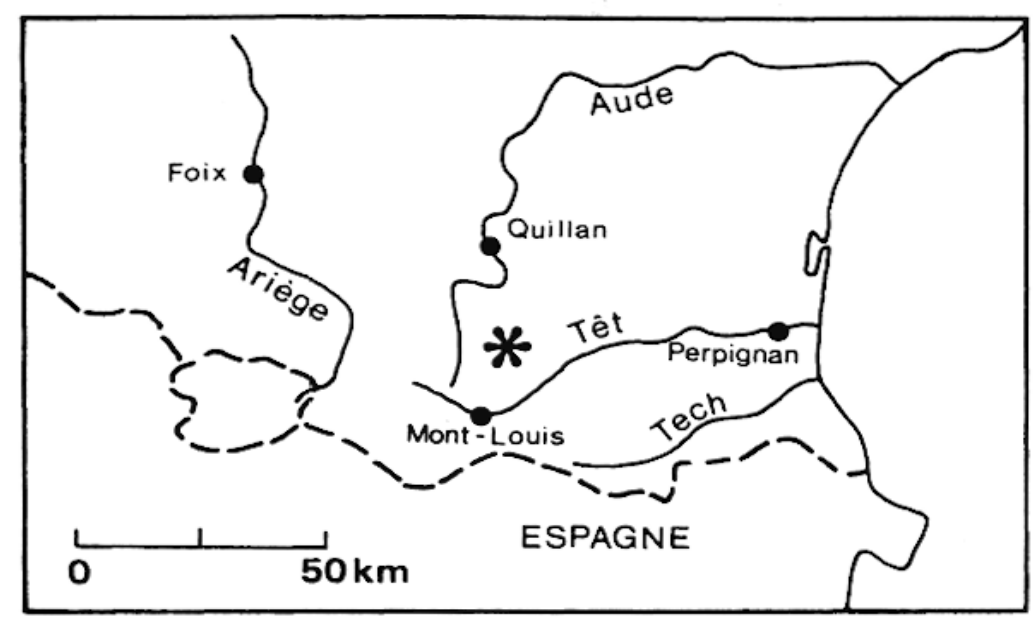

Fig. 1

Localisation des sîtes étudíss à Sansa, Pyrónées-Orientales.

Les quelques $23 \mathrm{~km}^{2}$ du territoire communal, situés entre 1150 et 2470 mètres d'altitude, sont couverts de forêts et de bois de pins à crochets, de pelouses alpines aux plus hautes altitudes ou, plus bas, de landes à genévrier commun ou à genêt purgatif. La topographie est variée, allant de " plas " sommitaux quasi horizontaux, situés vers $2300 \mathrm{~m}$ et échancrés par d'anciens cirques glaciaires, à de grands versants réguliers dont la dénivellation peut dépasser 1000 mètres.

Aucune terre n'est cultivée aujourd'hui dans la commune. Il n'en allait pas de même au siècle dernier : il n'est qu'à considérer la place occupée autour du village par les anciennes terrasses de culture, transformant les bas de versants en de véritables escaliers. Vers 1850, Sansa comptait 250 habitants. Les terrasses portaient des champs de seigle et chaque famille possédait un troupeau de quelques dizaines de moutons, soit environ 2000 têtes de petit bétail pour l'ensemble de la commune. Le gros bétail, constitué exclusive- 
ment d'animaux de trait, était peu nombreux. Face à ces effectifs, la charge pastorale actuelle, surtout ovine, apparait des plus modestes. De grandes parties du finage ne sont plus parcourues depuis une quarantaine d'années.

C'est notamment le cas de la soulane de Sansa, l'un de ces grands versants dont il est fait mention un peu plus haut, sorte de très vaste amphithéâtre ouvert au sud-ouest (fig. 2). La crête sommitale culmine à $2370 \mathrm{~m}$, au Pic de la Pelade. Le village, au pied du versant, est situé à $1400 \mathrm{~m}$. L'unité de cet ensemble tient autant à la monotonie et à la continuité des pentes, comprises entre 20 et 30 degrés, qu'à la faiblesse du couvert forestier : la soulane de Sansa est parsemée de petits bois de pins à crochets, dont la superficie cumulée ne représente pas $20 \%$ de la superficie totale du versant. A une exception près, dont nous ne traiterons pas, ces bois n'ont jamais été aménagés et soumis au régime forestier. C'est la dynamique de ces peuplements depuis la fin du $\mathrm{XIX}^{\circ}$ siècle que nous avons essayé de reconstituer.

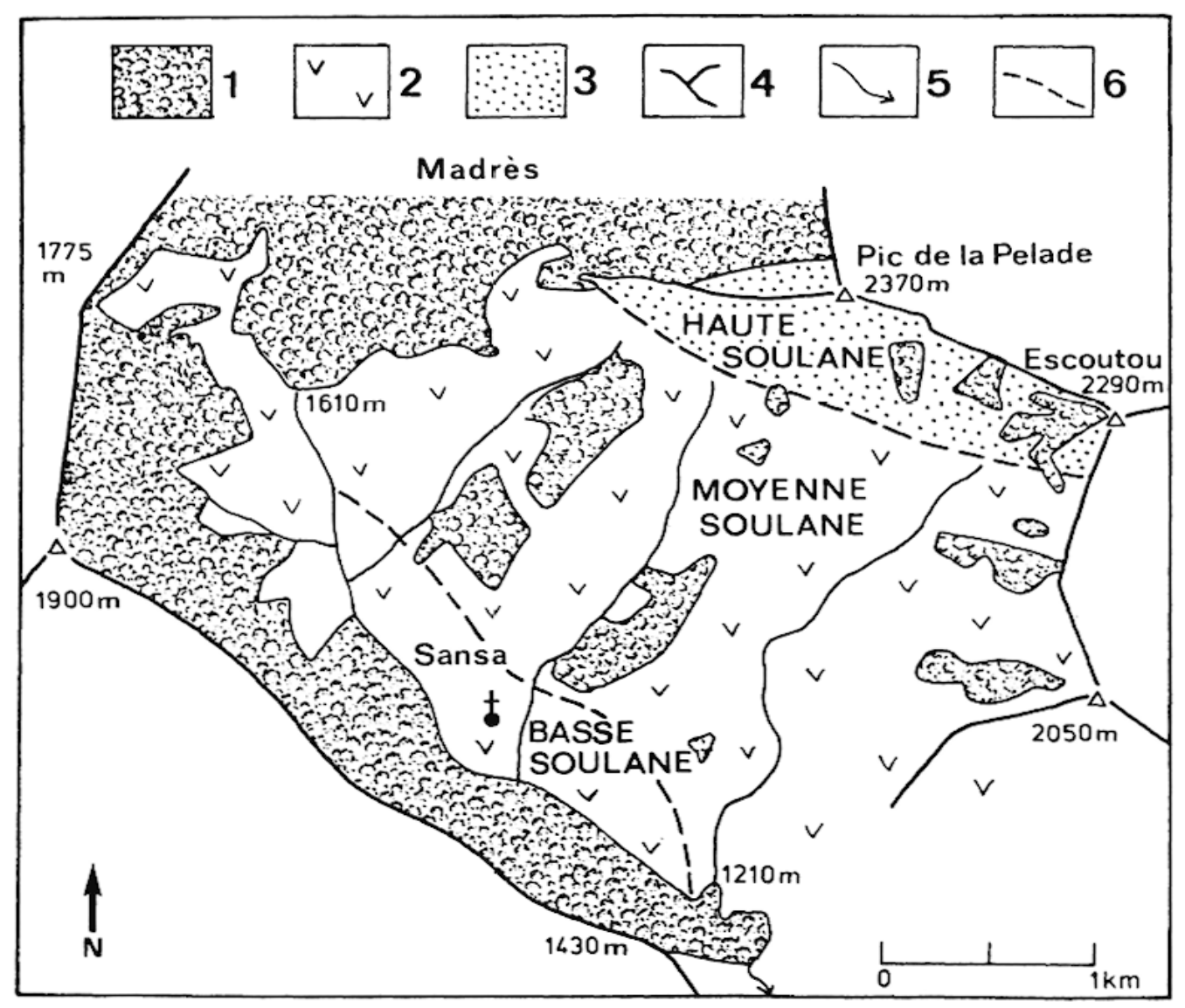

Fig. 2

La soulane de Sansa.

1. Forêt ou bois (pin à crochets majoritaire). - 2. Lande à genêt purgatif. 3. Pelouse calcicole et éboulis. - 4. Ligne de crête. - 5. Ruisseau, torrent. 6. Limite des "trois" soulanes (voir texte). 


\section{Une dynamique des peuplements forestiers changeante selon les lieux.}

La soulane de Sansa recoupe trois unités lithologiques distinctes. Dans la partie supérieure du versant affleurent des calcaires paléozoïques; dans la partie moyenne, des schistes; enfin, dans la partie inférieure, le granite du massif de Mont-Louis. Ce granite est assez fortement arénisé; des terrasses ont été aménagées au sein de cette formation meuble facile à mettre en culture. Un peu plus haut, entre 1600 et 2000 mètres, la "moyenne soulane schisteuse » est couverte d'une lande où domine le genêt purgatif, au milieu de laquelle apparaissent les premiers bois de pins à crochets. Jusqu'à la seconde guerre mondiale, cette lande était parcourue tous les ans par des feux pastoraux; elle ne l'est plus désormais que par des incendies d'origine accidentelle. Au-dessus de 2000 mètres et jusqu'à la crête sommitale s'étend la "haute soulane calcaire", bel exemple géomorphologique de versant réglé. De petits bois de pins à crochets y voisinent avec de vastes pierriers constitués de gélifracts, et avec une pelouse ouverte à faciès xérophile. Le sol se réduit ici à une rendzine très mince.

Comme on le voit, dans le détail, ce grand versant se présente comme une juxtaposition de «milieux » assez différents. D'où l'idée de comparer la dynamique historique des peuplements de pins en moyenne et en haute soulane.

\section{En haute soulane.}

Les bois de pins sont au nombre de trois, le plus vaste n'atteignant pas quinze hectares. Leur trait physionomique le plus remarquable est une répartition rigoureuse des arbres en quelques classes d'âge bien définies (fig. 3) : d'abord, une très large majorité d'arbres d'âge très homogène, compris entre 60 et 80 ans, en formation plus ou moins serrée; ensuite, disséminés parmi les précédents, et peu nombreux au total, de vieux pins séculaires; enfin, quelques jeunes arbres n'atteignant pas 40 ans, concentrés en lisière sud-est des trois bois, où ils forment une sorte de «front forestier pionnier " peu profond (20 ou 30 mètres tout au plus). Précisons qu'en ces lieux, la tramontane, vent de secteur nord-ouest, souffle très violemment. Les jeunes pins se sont peu développés ainsi au niveau des lisières «sous le vent ».

Il ressort évidemment de cet examen qu'une forte régénération du pin à crochet s'est produite en haute Soulane voici 60 à 80 années. Les porte-graines ne faisaient pas défaut, comme en témoigne la 


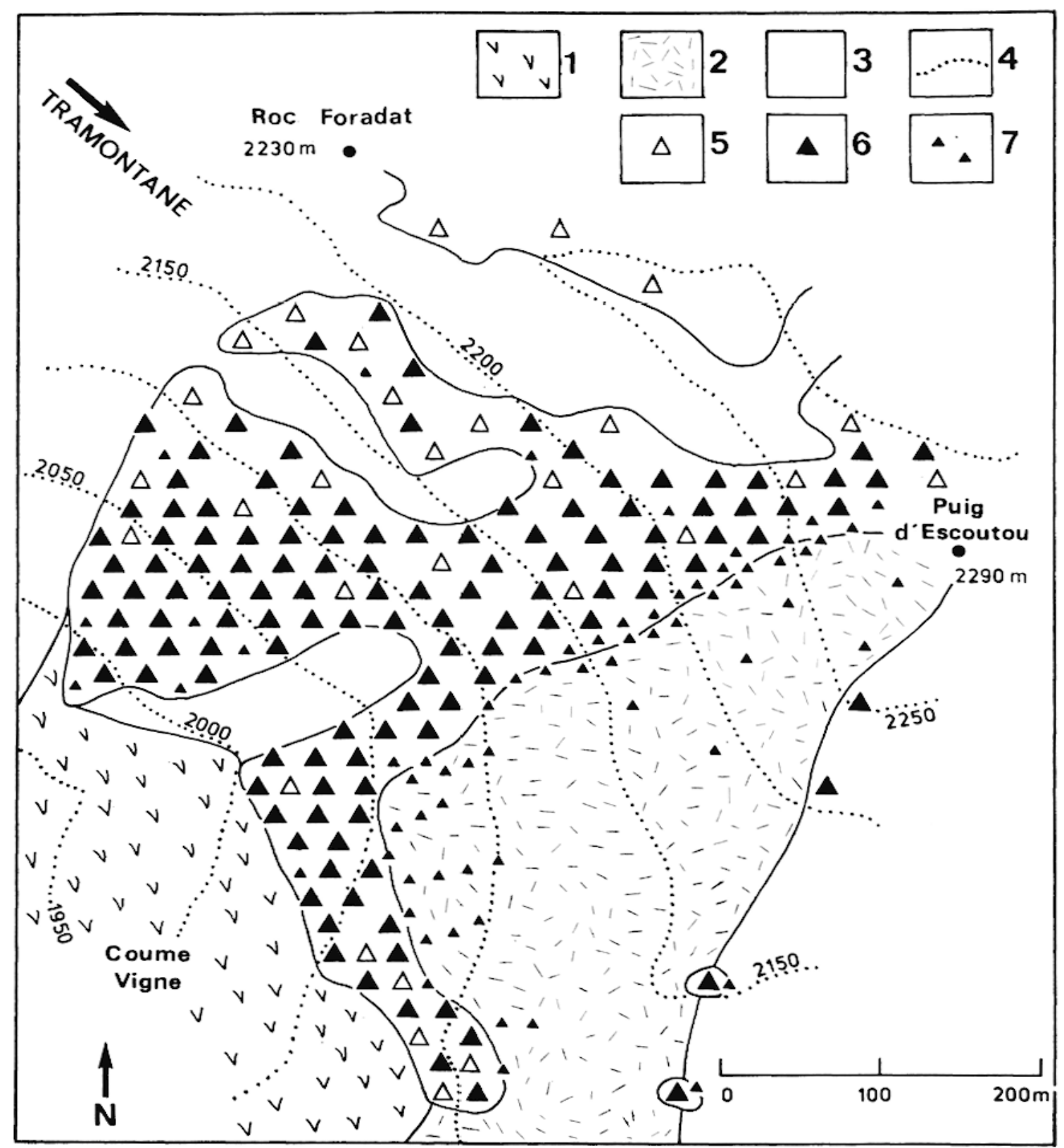

FIG. 3

Le bois du Puig d'Escoutou (haute soulane de Sansa) : physionomie actuelle.

1. Lande à genêt purgatif (moyenne soulane). - 2. Eboulis (gélifracts calcaires). - 3. Pelouse ouverte calcicole. - 4. Courbes de niveau (équidistance $50 \mathrm{~m}$ ). - 5. Pins âgés de plus d'un siècle. - 6. Pins âgés de $60 \mathrm{à} 80$ ans. - 7 . Pins âgés de moins de 40 ans.

présence actuelle de quelques arbres séculaires. En revanche, la tendance à l'extension des bois en direction du sud-est, qui semble se manifester depuis une quarantaine d'années, apparaît très limitée. La minceur des "fronts pionniers * l'atteste, aussi bien que la lecture 
attentive des photographies aériennes de ce secteur prises à différentes époques (les plus anciennes en 1942).

\section{En moyenne soulane.}

C'est la classe d'âge des individus de moins de 60 ans et surtout ce moins de 40 ans qui devient dans les bois de pins étudiés très nettement prédominante. Là encore, on note l'existence de quelques arbres séculaires au milieu de peuplements donc plus jeunes qu'en haute soulane. Les «fronts pionniers" sont absents : les jeunes pins sont présents en tous lieux. Il est vrai que la tramontane se fait moins tyrannique à ces altitudes plus basses.

L'évolution apparaît ici beaucoup plus simple: le processus de reboisement naturel, amorcé sans doute dans les années 1930, s'est poursuivi jusqu'à l'époque actuelle en s'accélérant après 1945. On est bien loin de la dynamique timide observée en haute soulane dans la période de l'après-guerre. Par contre, il est impossible de mettre en évidence une phase importante de régénération du pin voici 60 à 80 années, au début de ce siècle.

\section{Une combinaison de facteurs explicatifs d'ordre physique et socio-économique.}

La dynamique des peuplements forestiers de la moyenne soulane pose a priori le moins de difficultés d'interprétation. L'extension et la densification de ces peuplements, spectaculaires après la seconde guerre mondiale, peuvent être aisément corrélés avec le déclin des activités d'élevage dans la commune. La population de Sansa avait beaucoup décliné de 1900 à 1940, passant de 170 à moins de 60 habitants, mais l'effectif du troupeau communal demeurait élevé durant cette période, supérieur à 1000 têtes. Ce n'est qu'après 1945 que cet effectif se mit à fondre rapidement, et de façon irréversible.

En allant plus loin, sans doute faudrait-il évoquer, parallèlement à la diminution de la charge en bétail sur cette moyenne soulane, l'arrêt de la pratique séculaire de feux pastoraux. Ceux-ci devaient constituer un autre obstacle à la régénération des pins à crochets. L'intérêt des habitants de Sansa était d'ailleurs qu'il en soit ainsi : en empêchant que des arbres ne poussent sur un versant voué au pâturage, on évitait qu'il ne soit classé, au moins en partie, reforestable, c'est-à-dire que l'administration ne prenne des mesures pour y limiter le nombre de bêtes admises.

Il est plus délicat de rendre compte de la dynamique des peuplements forestiers de la haute soulane. Le paradoxe réside dans le fait que le "moment fort " de cette dynamique, l'importante phase de régénération du pin à crochets au début du siècle, se place bien 
avant le déclin des activités d'élevage dans la commune. Par contre, lorsque ce déclin se produit, après 1945 comme nous venons de le voir, la physionomie des bois de pins ne se modifie guère. Tout au plus s'étendent-ils très légèrement en direction du sud-est.

Nous proposons deux éléments d'explication à cet apparent paradoxc. En premier lieu, il est remarquable que la phase de régénération du début du siècle coïncide avec une série d'années très humides et exceptionnellement enneigées dans l'est des Pyrénées (tabl. 1). Cette conjoncture climatique pourrait avoir favorisé la régénération du pin dans le milieu édaphiquement très sec qu'est la haute soulane. A l'inverse, les années 1950 puis 1960 peu arrosées dans la région ne sont peut-être pas étrangères à la faible proportion de jeunes arbres dans les bois de la haute soulane.

\begin{tabular}{|c|c|c|}
\hline décennie & précipitations & températures \\
\hline $1872-1879$ & $830,4 \mathrm{~mm}$ & $7,1^{\circ} \mathrm{C}$ \\
$1880-1889$ & $810,2 \mathrm{~mm}$ & $6,6^{\circ} \mathrm{C}$ \\
$1890-1899$ & $821,4 \mathrm{~mm}$ & $7,2^{\circ} \mathrm{C}$ \\
$1900-1909$ & $906,7 \mathrm{~mm}$ & $6,8^{\circ} \mathrm{C}$ \\
$1910-1919$ & $974,5 \mathrm{~mm}$ & $6,5^{\circ} \mathrm{C}$ \\
$1921-1925 \in \mathrm{L} 1929$ & $703 \mathrm{~mm}$ & $7,1^{\circ} \mathrm{C}$ \\
$1930-1938$ & $830,5 \mathrm{~mm}$ \\
$1944-1949$ & $516,3 \mathrm{~mm}$ & \\
$1950-1959$ & $563,4 \mathrm{~mm}$ & \\
$1960-1969$ & $709,6 \mathrm{~mm}$ & \\
\hline
\end{tabular}

TABLEAU 1

TEMPERATURES ET PRECIPITATIONS RELEVÉES DE 1872 A 1969

A La STATION DE MONT-LouIs (1 $526 \mathrm{~m}$ ).

Moyennes décennales (sauf indication contraire, faute de données) des totaux pluviométriques annuels et des températures moyennes annuelles. Les valeurs furent respectivement de $1425,4 \mathrm{~mm}$ et $6,1^{\circ} \mathrm{C}$ en 1915 et de $1309,6 \mathrm{~mm}$ et $5,9^{\circ} \mathrm{C}$ en 1917 (Documents réunis par G. Casanobe et G. Soutadé).

L'examen de l'organisation du circuit pastoral traditionnel, qui réglait le déplacement des bêtes à l'intérieur du finage au cours de l'année, fournit une seconde explication. La haute soulane recevait le bétail à la fin de l'automne et au mois de mai. On ne pouvait y envoyer de bêtes du $1^{\text {er }}$ juin à la mi-octobre (1). L'altitude du versant

(1) L'usage en fut réglementé très tôt, puisqu'une délibération du Conseil municipal de Sansa du 7 Fructidor, an IX, " déclare devèse ou réserve depuis le 13 prairial de chaque année et pendant tout l'été et une partie de l'automne (...) toute cette partie du terroir appelée Coume Vigne ». (Archives départementales des P.O., série O). La Coume Vigne est le vallon dans lequel se situent les bois de la haute soulane. 
dépasse 2000 mètres, mais, en année normale, la bonne exposition devait garantir un libre accès aux ovins à ces deux périodes de l'année. Mais si l'hiver était précoce, si la neige se maintenait au-delà du $1^{\text {er }}$ juin, ces lieux se trouvaient accidentellement non parcourus par les troupeaux pendant plus d'un an. Il a dû en être ainsi à plusieurs reprises lors de ce début de siècle enneigé. Les jeunes pins échappaient alors aux dégradations des troupeaux.

\section{Conclusion}

C'est donc sur une hiérarchie différente des facteurs explicatifs que s'opposent les dynamiques forestières en moyenne et en haute soulane de Sansa. Le schéma entrevu en moyenne soulane est conforme au modèle le plus répandu, à savoir que l'extension de l'arbre dans les communes de montagne touchées par l'exode rural est d'abord consécutif au ralentissement des activités pastorales. En haute soulane, la dynamique forestière paraît beaucoup plus inféodée au climat, qu'il faille rendre compte des épisodes de régénération du pin à crochets ou de l'extension des bois conformément à la direction de la tramontane. Il doit donc exister un seuil, dépendant entre autres de l'altitude, exprimant ce renversement de l'ordre des facteurs explicatifs : socio-économiques et climatiques. Les deux se combinent de toutes les façons, avec certainement plusieurs facteurs que nous n'avons pu évoquer ici. Ces recherches en appellent d'autres...

\section{Bibliographie}

La limite supérieure de la forêt et sa valeur de seuil. Colloque de Perpignan, 1981. Centre de Perpignan de l'Institut d'Etudes andorranes.

Bécat (J.), 1974. - La vallée d'Evol. Vivre en Haut Conflent. Revue Conflent, Prades.

DESAILLY (B.), 1984. - Recherches géographiques sur les paysages et leur dynamique dans le massif du Madrès. Secteur de Sansa (P.O.). Mém. de Maîtrise, Université de Paris $\mathrm{X}$ - Nanterre.

MARCEL (J.-F.), 1982. - Recherches sur les communautés orophytiques du massif du Madrès. Applications a la dynamique des versants et à la limite supérieure de la forêt. Thèse $3^{\circ}$ cycle, Université Paul Sabatier, Toulouse. 
Résumé. - La soulane de Sansa (Pyrénées-Orientales) présente, entre $1400 \mathrm{~m}$ et $2370 \mathrm{~m}$, une juxtaposition de milieux à dynamiques forestières différentes, dans le temps comme dans l'espace. La combinaison de facteurs physiques (histoire du climat) et humains (pratiques pastorales) permet d'expliquer les variations dans le repeuplement forestier.

Summary. - Pastoral abandonment and tree-Growing dynamics. The Sansa « soulane $*$ (south-facing slope) in the Pyrénées-Orientales, displays, between the altitudes of $1400 \mathrm{~m}$ and $2370 \mathrm{~m}$, a juxtaposition of environment with different forest dynamics in both time and space. The combination of physical factors (climatic history) and human factors (pastoral farming methods) gives an explanation of the changing pattern of reforestation.

RESUMen. - Retroceso del PASTOREO Y diNAMiCa de las poblaciones forestales. La solana de Sansa (Pyrénées-Orientales) presenta, entre 1400 y $2370 \mathrm{~m}$, una yuxtaposición de medios naturales caracterizados por dinámicas forestales diversas, tanto en el tiempo como en el espacio. La combinación de factores físicos (historia del clima) y humanos (prácticas pastorales) permiten entender las variaciones observables en la repoblación forestal.

Mots-Clés. - Pyrénées, Pyrénées-Orientalcs, Madrès, Dynamique forestière, exploitation pastorale, soulane, Pin à crochets. 